\title{
AVALIAÇÃO DE CULTIVARES E HIBRIDOS DE BANANEIRA EM LAVRAS, MG ${ }^{1}$
}

\author{
LAIR VICTOR PEREIRA ${ }^{2}$ \\ SEBASTIÃO DE OLIVEIRA E SILVA ${ }^{3}$ \\ ELIO JOSÉ ALVES \\ CARLOS RAMIREZ DE REZENDE E SILVA ${ }^{5}$
}

\begin{abstract}
RESUMO - Avaliaram-se os genótipos de bananeira Nam, Pioneira, Caipira, Prata Anã, Grande Naine, SH36-40, FHIA 18, FHIA 01 e PV 03-44, em condições de sequeiro na região sul de Minas Gerais, com o objetivo de identificar os mais produtivos e com melhor adaptação edafoclimática, em comparação com as cultivares Prata e Prata Anã, tradicionalmente cultivadas nessa região. O ensaio foi conduzido na Fazenda Experimental da EPAMIG, em Lavras, no período de 1997 a 1999. Cada genótipo foi plantado em blocos de 50 plantas, no espaçamento $3,0 \times 3,0 \mathrm{~m}$, sendo a parcela útil as 24 plantas centrais e uma por repetição. O híbrido SH36-40 foi superior aos demais em peso médio dos cachos $(16,56 \mathrm{~kg})$, frutos $(171,00 \mathrm{~g})$, diâmetro $(4,21$ $\mathrm{cm})$ e comprimento dos frutos $(17,64 \mathrm{~cm})$ nos três primeiros ciclos. Isso corresponde a um rendimento de
\end{abstract}

TERMOS PARA INDEXAÇÃO: Musa spp., banana, variedades, produção e ciclo da cultura.

18,5 t/ha, cerca de duas vezes maior que a produção das cultivares Prata Anã, Pioneira, Nam, Caipira e três vezes maior que a do híbrido PV 03-44. O híbrido SH 3640 apresentou, ainda, porte médio de $2,75 \mathrm{~m}$, ciclo em torno de 17,5 meses e o menor período da emissão à colheita do cacho, inferior a 4,83 meses para as plantas-mãe, filha e neta. Esse híbrido apresentou também um menor e mais tardio perfilhamento. Os híbridos SH 36-40 e FHIA 01 apresentaram os maiores incrementos nos pesos dos cachos e dos frutos do primeiro para o terceiro ciclo. As cultivares Caipira, Prata Anã, Pioneira e Nam produziram cachos com peso médio inferior a 9,0 kg e o híbrido PV0344, inferior a $6,0 \mathrm{~kg}$. A cv. Caipira foi a mais tardia, com um ciclo em torno de 22 meses e a Prata Anã e Pioneira, as mais precoces, com cerca de 16,6 meses no primeiro ciclo e 15,5 no segundo e terceiro ciclos.

\section{BANANA HYBRIDS AND CULTIVAR EVALUATION IN LAVRAS-MG}

\begin{abstract}
The banana genotypes Nam, Caipira, Pioneira, Prata anã, Grande Naine, FHIA 01, FHIA 18, SH 36-44, and PV 03-44 were evaluated from 1997 to 1999. The objective was to identify the most productive and most adaptated genotype compared to the traditional Prata and Prata-Anã cultivars in the southern State of Minas Gerais, Brazil. The experiment was established in the EPAMIG Experimental Station in Lavras-MG. Fifty plants of each genotype were planted in a 3,0 $\times 3,0 \mathrm{~m}$ plant spacing. The $\mathrm{SH} 36-40$ hybrid presented the greater bunch medium weight $(16.56 \mathrm{~kg})$
\end{abstract}

and fruits (171 g/unit), diameter $(4.21 \mathrm{~cm})$ and length $(17.64 \mathrm{~cm})$ of the fruits. It is equivalent to a yield of $18.5 \mathrm{t} / \mathrm{ha}$, which is about twice the yield of the Prata Anã, Pioneira, Caipira, and Nam, and thrice the yield of PV 03-44. SH 36-40 and FHIA 01 hybrids presented the greatest increases in bunches and fruits weight from one cycle to the next. Nam produced bunches with medium weight inferior to $9.0 \mathrm{~kg}$, and the PV 03-44 hybrid produced $6.0 \mathrm{~kg}$. The hybrid $\mathrm{SH} 36-40$ showed plant hight of $2.75 \mathrm{~m}$, crop cycle of 17.5 months, the smallest period from flowering to harvesting, inferior

1. Trabalho financiado pela EPAMIG/CTSM.

2. Engenheiro Agrônomo, MSc., Pesquisador da Epamig, Caixa Postal 176, 37200-000 - Lavras, MG, Epamig @ufla.br

3. Engenheiro Agrônomo, PhD., Pesquisador da Embrapa Mandioca e Fruticultura, Caixa Postal 007, 44380-000 Cruz das Almas, BA, silva@cnpmf.embrapa.br

4. Engenheiro Agrônomo, MSc., Pesquisador da Embrapa Mandioca e Fruticultura. elio@cnpmf.embrapa.br

5. Engenheiro Agrônomo, MSc., Professor do Departamento de Agricultura da UNIVERSIDADE FEDERAL DE LAVRAS/UFLA, Caixa Postal 37, 37200-000 - Lavras, MG, dag@ufla.br 
to 4,87 months, and smaller and later sprouting. The cultivars Caipira, Prata Anã, Pioneira, and Nam produced bunches with medium weight inferior to 9.0 $\mathrm{kg}$ and the PV 03-44 hybrid inferior to $6.0 \mathrm{~kg}$. The
Caipira was the tardiest cultivar, with 22.0 month of crop cycle, and the cultivars Pioneira and Prata Anã were the most precocious, 16.6 months, in the first cycle and 15.5 months in the second and third cycles.

INDEX TERMS: Musa spp., banana, yield, cultivar, crop cycle.

\section{INTRODUÇÃO}

A cultura da bananeira tem-se expandido bastante nos últimos anos, tanto em Minas Gerais como em outros Estados do Brasil. Apesar da melhoria do nível tecnológico, para o seu cultivo em algumas regiões, o rendimento ou produtividade da bananicultura brasileira ainda é muito baixo. Segundo FrutiSeries (2000), em 1999, o Brasil foi o terceiro maior produtor de banana, com 5,6 milhões de toneladas em uma área plantada de 518.000 ha. Isso corresponde a um rendimento de 10,81t/ha.

Em Minas Gerais, o rendimento da cultura da banana em 1999 foi de 10,2 t/ha (FrutiSéries, 2000). Há, ainda, grande diferença no rendimento da cultura entre as regiões produtoras de banana do Estado de Minas Gerais, em razão principalmente do nível tecnológico empregado, incentivos governamentais exclusivos para algumas regiões e cultivar explorada. $\mathrm{O}$ rendimento e a qualidade da banana dependem do nível tecnológico de cultivo, manejo, incidência e controle de pragas e doenças, condições edafoclimáticas favoráveis e características inerentes à cultivar (Alves et al. 1999). É importante salientar que somente a resistência a pragas e doenças e rendimento de uma cultivar não são suficientes para determinar o sucesso, em termos de sua adoção pelos bananicultores. A substituição de uma cultivar tradicionalmente explorada numa ou várias regiões do mundo, em virtude de sua maior aceitação no mercado, é difícil, pois, a banana é uma fruta de consumidor exigente em sabor (Silva \& Alves, 1999). Segundo Orjeda et al., (1999), uma nova variedade de bananeira para ser bem aceita pelos produtores e mercado consumidor precisa combinar resistência ou tolerância a pragas e doenças, alta produtividade e frutos com boas características póscolheita e organolépticas.

A cv. Prata, apesar de suscetível à Sigatoka amarela e ao Mal do Panamá e apresentar baixo rendimento, é a mais cultivada na região sul de Minas Gerais, por ser a preferida pelo consumidor.

Silva \& Alves (1999) observaram que dentre 18 genótipos de banana avaliados nas condições da Bahia, as cultivares Caipira e Grande Naine e os híbridos FHIA 01, FHIA 18 foram os mais produtivos. Por outro lado, o 'FHIA 18' e a 'Pioneira' apresentaram ciclo vegetativo menor que 12 meses, ao passo que para 'FHIA 01' e 'Prata Anã', o ciclo foi maior que 13,5 meses. Pereira et al. (1998) afirmaram que as cultivares Prata, Prata Anã e Mysore foram mais produtivas em Maria da Fé e Lavras, região sul de Minas, do que em Patrocínio, região do Triângulo Mineiro/Alto Paranaíba, e a cv. Prata Anã, em Maria da Fé, produziu cachos significativamente maiores que em Lavras, ocorrendo o contrário com a cv. Mysore. Segundo esses autores, o ciclo dessas cultivares em Maria da Fé foi cerca de 4,21 meses maior que em Lavras e Patrocínio. Souto et al. (1996) observaram que na região norte de Minas o híbrido PV03-44 foi mais precoce e a cv.Thap Maeo mais produtiva do que as cultivares Pacovan, Ouro da Mata, Mysore, Nam e Caipira. Silveira et al. (1996), avaliando o comportamento de cultivares e híbridos de bananeira nessa mesma região, constataram que os híbridos PA 03-22 e a cv. Pioneira apresentaram maior produção e precocidade que a Prata Anã. Por outro lado, Gomez \& Nobrega (2000) verificaram que na região produtora de banana do Estado do Espírito Santo a cv. Thap Maeo foi mais produtiva e a cv. Pioneira a mais precoce que as cultivares Prata, Mysore, Caipira e o híbrido PV 03-44. Ganga et al. (2001) também observaram que as cultivares Pioneira e Prata Anã foram mais precoces no florescimento que os híbridos FHIA 01 e FHIA 18 e a cv. Maça, em Jaboticabal-SP.

Resultados de outros trabalhos realizados nos projetos irrigados do Jaíba e Gorutuba, região norte de Minas Gerais, infere-se que os híbridos FHIA 01, FHIA 18 e SH 36-40 produziram cachos com peso duas vezes maior que o das cultivares Prata Anã e Pioneira e 1,5 vez maior que a cv. Caipira (Gonçalves et al., 2001; Souto et al., 2001 e Rodrigues et al., 2001). Quanto ao ciclo da cultura, as cultivares Prata Anã e Pioneira foram as mais precoces, com 310 dias, ao passo que o híbrido $\mathrm{SH} 36-40$ e a cv. Caipira foram as mais tardias, com 370 e 382 dias, respectivamente (Gonçalves et al., 2001).

Segundo Alvarez (1997), em Cuba, o híbrido FHIA 01, nos dois primeiros ciclos, apresentou rendimento duas vezes maior (50t/ha) que a cv. Grande Naine (24 t/ha). Navarro \& Caicedo (1998), na Colom- 
bia, também verificaram que os híbridos FHIA 01 e FHIA 02 com rendimento de $27 \mathrm{t} /$ ha foram cerca de 2,4 vezes mais produtivos que a cv. Grande Naine.

Segundo Cerqueira et al. (2001), em Cruz das Almas, BA, o híbrido SH 36-40 produziu frutos maiores que os demais avaliados, entre eles, o 'PV 03-44' e as cultivares, Prata, Nam, Prata Anã e Pacovam.

Com relação ao perfilhamento, Souza et al. (1996), Lameira et al. (1998) e Dantas et al. (1986) concluíram que há diferença na capacidade de proliferação de cada genótipo, afetando, assim, a produção de rebentos.

Com este trabalho objetivou-se avaliar o comportamento vegetativo e produtivo de nove genótipos de bananeira, identificando os mais produtivos e com melhor adaptação às condições edafoclimáticas da região sul de Minas Gerais, visando a sua incorporação ao sistema de produção dessa região.

\section{MATERIAL E MÉTODOS}

O experimento foi conduzido na Fazenda Experimental da EPAMIG, em LAVRAS, no período de maio de 1997 a outubro de 1999.

O solo da área experimental foi classificado como uma associação de Podzólico Vermelho-Escuro, Distrófico-epieutrófico, textura muito argilosa, mais Terra Roxa Estruturada-Eutrófico, textura Argilo$\mathrm{sa} /$ muito argilosa.

Lavras está a $855 \mathrm{~m}$ de altitude e $21^{\circ} 15^{\prime} 00^{\prime}$ de latitude sul. O clima da região é subtropical. As médias das temperaturas nos períodos de condução do experimento foram: das mínimas, $15,3^{\circ} \mathrm{C}$, com amplitude térmica de 7,0 a $19,2^{\circ} \mathrm{C}$; das máximas de $27,7^{\circ} \mathrm{C}$, com amplitude térmica de 23,7 a $30,0^{\circ} \mathrm{C}$ e média geral de $20,4^{\circ} \mathrm{C}$. A precipitação anual foi de $1.380 \mathrm{~mm}$, e de maio a agosto, período seco e frio, ocorreram dois meses com menos de $60 \mathrm{~mm}$ de chuva/mês.

Foram utilizadas mudas de cultura de meristema, inicialmente plantadas em sacos de polietileno contendo 4,0 1 da mistura solo, esterco e areia na proporção 2:1:1. Nesses recipientes, as mudas permaneceram em telado sob irrigação e adubação controladas durante dois meses, para aclimatação e aceleração do desenvolvimento. O transplantio para o campo ocorreu em 05/05/97, sendo o experimento mantido sob irrigação por aspersão, duas vezes por semana até final de agosto. A partir daí, o experimento foi conduzido em condição de sequeiro.

Foram avaliados os seguintes genótipos: Pioneira, SH 36-40, FHIA 01 e FHIA 18 (AAAB, híbridos de Prata Anã), PV 03-44 (AAAB, híbrido de Pacovan), Caipira e Nam (AAA, cultivar sem subgrupo), Prata
Anã (AAB, cultivar tipo Prata) e Grande Naine (AAA, cultivar tipo Cavendish). As duas últimas cultivares foram incluídas para servir de comparação, por serem tradicionalmente cultivadas no Brasil e pela semelhança de sabor com as cultivares e híbridos avaliados.

O bananal foi conduzido no sistema família, por meio de desbastes, deixando-se apenas um seguidor por geração.

Cada genótipo foi plantado em bloco único de 50 plantas no espaçamento 3,0 x 3,0 m, sendo considerada como parcela útil as 24 plantas centrais. Para efeito de análise estatística, cada planta constituiu uma repetição.

A correção da fertilidade e acidez do solo foi feita com base nos resultados da análise do solo e exigências nutricionais da cultura, conforme recomendações da Comissão... (1989).

Não foi realizado o controle químico de pragas e doenças, ao passo que o de plantas daninhas foi feito alternando-se capinas manual e química à base de Paraquat.

O comportamento vegetativo da planta-matriz, do primeiro rebento (filho) e do segundo rebento (neto) foi avaliado semanalmente por meio das seguintes variáveis: período decorrido para emissão dos primeiros filhos e netos, número de filhos e de netos emitidos até a época da emissão do cacho, altura da planta (ao nível da roseta foliar), diâmetro do pseudocaule a $40 \mathrm{~cm}$ do nível do solo na época da emissão do cacho, e ciclo da cultura subdividido em períodos de pré-floração (do plantio à emissão do cacho), de frutificação (da emissão à colheita do cacho) e total (do plantio à colheita do cacho).

A produção foi avaliada por meio do peso do cacho e do fruto, comprimento e diâmetro do fruto central da penca mediana do cacho e número de frutos por cacho. As avaliações foram realizadas até o final da colheita dos cachos do terceiro ciclo (planta-neta).

Os dados foram submetidos à análise de variância e as médias comparadas por meio do teste de médias de Scott-Knott pelo soft-ware SISVAR (Ferreira, 2000).

\section{RESULTADOS E DISCUSSÃO}

Pelos dados da Tabela 1, verifica-se que o híbrido PV 03-44 e a cv. Caipira apresentaram na época da emissão do cacho maiores portes ou altura da planta, isto é, acima de $3,10 \mathrm{~m}$ no primeiro ciclo, de $3,70 \mathrm{~m}$ no segundo e de 4,10 m no terceiro, com incremento médio em torno de 0,5 m de um ciclo para o outro. Esses dois genótipos apresentaram também os menores diâmetros nos três ciclos avaliados, ou seja, inferiores a $16,0 \mathrm{~cm}$. A cv. Grande Naine apresentou menor porte, inferior a 
2,25 m nos três ciclos. Os demais genótipos apresentaram porte médio, os quais variaram de 2,40 a 2,66 m no primeiro ciclo, de 2,50 a 2,85 $\mathrm{m}$ no segundo e de 2,68 a $2,98 \mathrm{~m}$ no terceiro, com incremento médio no porte inferior a $0,20 \mathrm{~m}$, de um ciclo para o outro. Os híbridos SH 36-40, FHIA 01, FHIA 18 e a cv. Grande Naine apresentaram diâmetro do pseudocaule acima de $20,0 \mathrm{~cm}$ nos três ciclos, com destaque para o híbrido SH 36-40, com 22,90; 24,06 e 25,86 cm nos primeiro, segundo e terceiro ciclos, respectivamente.

Com relação ao perfilhamento (Tabela 2), a cv. Caipira foi a mais precoce, emitindo os primeiros filhos aos 3 meses e netos aos 6,2 meses, após o plantio. Essa cultivar apresentou também um perfilhamento mais intenso: 5,7 filhos e 10,50 netos até a época da emissão do cacho. O híbrido SH 36-40 foi o mais tardio, com emissão dos primeiros filhos aos 4,4 e dos netos aos 8,7 meses após o plantio e produziu o menor número de filhos $(3,10)$ e de netos $(6,00)$ até a época da emissão do cacho. Os demais genótipos emitiram os primeiros filhos em torno de 3,6 meses e netos aos 7,3 meses, após o plantio, com uma produção em torno de 3,5 filhos e 7,2 netos até a época da emissão do cacho.

O híbrido SH 36-40 produziu cachos e frutos significativamente maiores que os demais genótipos nos três ciclos avaliados (Tabela 3), e somente no primeiro ciclo a cv. Grande Naine produziu cacho com peso e- quiparado ao daquele híbrido $(14,636 \mathrm{Kg})$. Em relação ao peso e tamanho dos frutos (Tabela 4), o híbrido $\mathrm{SH}$ 36-40 foi significativamente superior aos demais genótipos, produzindo frutos com peso médio de 157,10; 172,02 e 183,88 g e diâmetro x comprimento de 4,16 x 16,$86 ; 4,22$ x 17,84 e 4,26 x 18,21 cm, respectivamente no primeiro, segundo e terceiro ciclos. Considerando a evolução da produção do primeiro para o terceiro ciclo, observa-se que o híbrido SH 36-40 apresentou o maior incremento no peso do cacho $(25,67 \%)$, seguido pelo híbrido FHIA 01, com $20,56 \%$ e pela cv. Nam, com $12,99 \%$ (Tabela 3). Considerando-se ainda a produtividade média dos três ciclos, o híbrido SH 36-40 foi o que apresentou maior rendimento, ou seja, 18,50 t/ha.

O híbrido PV 03-44 foi inferior aos demais genótipos nos três ciclos avaliados, produzindo cachos com peso médio inferior a $6,0 \mathrm{~kg}$ e com menos de 73 frutos (Tabela 3). Por outro lado, a cv. Caipira, apesar de ter produzido cachos com mais de 100 frutos (Tabela $3)$, apresentou menor peso $(72,0 \mathrm{~g})$, comprimento $(12,2$ $\mathrm{cm})$ e diâmetro $(3,2 \mathrm{~cm})$ (Tabela 4). Somente os híbridos SH 36-40 e FHIA 01 e a cv. Nam tiveram aumento no tamanho dos frutos, do primeiro para o terceiro ciclo, destacando-se novamente o híbrido SH 36-40, com $17 \%$ no peso, $2,4 \%$ no diâmetro e $8,0 \%$ no comprimento (Tabela 4).

TABELA 1 - Altura da planta e diâmetro do pseudocaule de cultivares e híbridos de bananeira nos três primeiros ciclos, Lavras, MG, 2000.

\begin{tabular}{lcccccc}
\hline \multirow{2}{*}{$\begin{array}{c}\text { Cultiva- } \\
\text { res/Híbridos }\end{array}$} & \multicolumn{3}{c}{ Altura (m) } & \multicolumn{3}{c}{ Diâmetro (cm) } \\
\cline { 2 - 7 } & $\mathbf{1}^{\mathbf{0}}$ Ciclo & $\mathbf{2}^{\mathbf{0}}$ Ciclo & $\mathbf{3}^{\mathbf{0}}$ Ciclo & $\mathbf{1}^{\mathbf{0}}$ Ciclo & $\mathbf{2}^{\mathbf{0}}$ Ciclo & $\mathbf{3}^{\mathbf{0}}$ Ciclo \\
\hline PV 03-44 & $3,38 \mathrm{a}$ & $3,89 \mathrm{a}$ & $4,40 \mathrm{a}$ & $15,34 \mathrm{f}$ & $15,63 \mathrm{e}$ & $15,93 \mathrm{~h}$ \\
Caipira & $3,15 \mathrm{~b}$ & $3,73 \mathrm{~b}$ & $4,15 \mathrm{~b}$ & $15,21 \mathrm{f}$ & $15,41 \mathrm{e}$ & $15,59 \mathrm{i}$ \\
FHIA 18 & $2,66 \mathrm{c}$ & $2,86 \mathrm{c}$ & $2,98 \mathrm{c}$ & $20,30 \mathrm{c}$ & $21,70 \mathrm{c}$ & $20,60 \mathrm{~d}$ \\
FHIA 01 & $2,65 \mathrm{c}$ & $2,80 \mathrm{c}$ & $2,94 \mathrm{c}$ & $21,01 \mathrm{~b}$ & $29,71 \mathrm{~b}$ & $23,64 \mathrm{~b}$ \\
SH 36-40 & $2,60 \mathrm{c}$ & $2,75 \mathrm{c}$ & $2,85 \mathrm{~d}$ & $22,91 \mathrm{a}$ & $24,06 \mathrm{a}$ & $25,86 \mathrm{a}$ \\
Nam & $2,45 \mathrm{~d}$ & $2,58 \mathrm{~d}$ & $2,73 \mathrm{e}$ & $17,56 \mathrm{e}$ & $18,50 \mathrm{~d}$ & $18,41 \mathrm{e}$ \\
Pioneira & $2,42 \mathrm{~d}$ & $2,57 \mathrm{~d}$ & $2,70 \mathrm{e}$ & $17,87 \mathrm{~d}$ & $18,85 \mathrm{~d}$ & $18,15 \mathrm{f}$ \\
Prata anã & $2,40 \mathrm{~d}$ & $2,52 \mathrm{~d}$ & $2,68 \mathrm{e}$ & $17,82 \mathrm{~d}$ & $18,64 \mathrm{~d}$ & $17,84 \mathrm{~g}$ \\
Grande Naine & $2,05 \mathrm{e}$ & $2,15 \mathrm{e}$ & $2,22 \mathrm{f}$ & $20,23 \mathrm{c}$ & $22,13 \mathrm{~b}$ & $21,04 \mathrm{c}$ \\
\hline CV & $7,22 \%$ & $8,31 \%$ & $6,42 \%$ & $6,73 \%$ & $7,18 \%$ & $8,24 \%$ \\
\hline
\end{tabular}

Médias seguidas da mesma letra, na coluna, não diferem entre si pelo teste de SCOTT-KNOTT $($ P $\leq 0,05)$ 
TABELA 2 - Quantidade e período da emissão dos primeiros filhos e netos de cultivares e híbridos de bananeira, Lavras, MG, 2000.

\begin{tabular}{lcclc}
\hline \multirow{2}{*}{ Cultivares/Híbridos } & \multicolumn{2}{c}{ Quantidade $^{\mathbf{1}}$} & \multicolumn{2}{c}{ Época de emissão } \\
\cline { 2 - 5 } & Filhos & Netos & Filhos & Netos \\
\hline Caipira & $5,70 \mathrm{a}$ & $10,50 \mathrm{a}$ & $3,08 \mathrm{e}$ & $7,20 \mathrm{f}$ \\
Prata Anã & $5,25 \mathrm{~b}$ & $8,53 \mathrm{~b}$ & $3,10 \mathrm{e}$ & $7,35 \mathrm{f}$ \\
Pioneira & $5,01 \mathrm{c}$ & $8,06 \mathrm{c}$ & $3,40 \mathrm{~d}$ & $8,11 \mathrm{e}$ \\
FHIA 18 & $4,91 \mathrm{c}$ & $7,91 \mathrm{~d}$ & $3,88 \mathrm{c}$ & $8,66 \mathrm{c}$ \\
PV 03-44 & $4,88 \mathrm{c}$ & $7,81 \mathrm{~d}$ & $3,56 \mathrm{~d}$ & $8,43 \mathrm{~d}$ \\
Grande Naine & $4,86 \mathrm{c}$ & $7,50 \mathrm{e}$ & $3,68 \mathrm{c}$ & $8,63 \mathrm{c}$ \\
FHIA 01 & $4,81 \mathrm{c}$ & $7,45 \mathrm{e}$ & $3,91 \mathrm{~b}$ & $8,80 \mathrm{c}$ \\
Nam & $4,25 \mathrm{~d}$ & $7,01 \mathrm{f}$ & $4,13 \mathrm{a}$ & $9,36 \mathrm{~b}$ \\
SH 36-40 & $3,10 \mathrm{f}$ & $6,03 \mathrm{~g}$ & $4,28 \mathrm{a}$ & $9,60 \mathrm{a}$ \\
\hline CV & $3,80 \%$ & $2,89 \%$ & $4,49 \%$ & $2,99 \%$ \\
\hline
\end{tabular}

Médias seguidas da mesma letra, na coluna, não diferem entre si pelo teste de SCOTT-KNOTT (P $\leq 0,01)$.

${ }^{1}$ Número de filhos e netos emitidos até a época da emissão do cacho.

TABELA 3 - Valores médios de peso do cacho e número de frutos por cacho dos três primeiros ciclos de cultivares e híbridos de bananeira, Lavras, MG., 2000

\begin{tabular}{|c|c|c|c|c|c|c|c|c|}
\hline \multirow{2}{*}{$\begin{array}{l}\text { Cultivares/ } \\
\text { Híbridos }\end{array}$} & \multicolumn{3}{|c|}{ Peso dos Cachos (kg) } & \multirow{2}{*}{$\begin{array}{l}\text { Incremento } \\
\text { do } 1^{\circ} \text { para o } \\
3^{\circ} \text { Ciclo }(\%)\end{array}$} & \multicolumn{3}{|c|}{ Número de Frutos/Cacho } & \multirow{2}{*}{$\begin{array}{l}\text { Incremento } \\
\text { do } 1^{\circ} \text { para o } \\
3^{\circ} \text { Ciclo (\%) }\end{array}$} \\
\hline & $1^{\circ}$ Ciclo & $2^{\circ}$ Ciclo & $3^{\circ}$ Ciclo & & $1^{\circ}$ Ciclo & $2^{\circ}$ Ciclo & $3^{\circ}$ Ciclo & \\
\hline SH $36-40$ & $14,670 \mathrm{a}$ & $16,580 \mathrm{a}$ & 18,436 a & $+25,67$ & $93,38 \mathrm{c}$ & $96,38 \mathrm{~d}$ & $100,26 \mathrm{c}$ & +6.90 \\
\hline Grande Naine & $14,636 \mathrm{a}$ & $13,853 \mathrm{~b}$ & $12,792 \mathrm{c}$ & $-14,41$ & $114,20 \mathrm{a}$ & $109,30 \mathrm{a}$ & $106,20 \mathrm{a}$ & -7.53 \\
\hline FHIA 01 & $12,325 \mathrm{~b}$ & $13,350 \mathrm{~b}$ & $14,860 \mathrm{~b}$ & $+20,56$ & $96,06 \mathrm{c}$ & $99,10 \mathrm{c}$ & $103,25 \mathrm{~b}$ & +7.48 \\
\hline FHIA 18 & $10,596 \mathrm{c}$ & $10,640 \mathrm{c}$ & $10,640 \mathrm{~d}$ & $+0,41$ & $92,38 \mathrm{c}$ & $93,36 \mathrm{~d}$ & $93,30 \mathrm{~d}$ & +1.13 \\
\hline Prata anã & $8,622 \mathrm{~d}$ & $8,786 \mathrm{~d}$ & $8,282 \mathrm{f}$ & - 4,10 & $92,38 \mathrm{c}$ & $92,42 \mathrm{~d}$ & $91,26 \mathrm{e}$ & -1.24 \\
\hline Nam & $7,830 \mathrm{e}$ & $8,323 \mathrm{~d}$ & $8,836 \mathrm{e}$ & $+12,84$ & $86,26 \mathrm{~d}$ & $88,32 \mathrm{e}$ & $91,15 \mathrm{e}$ & +5.66 \\
\hline Pioneira & $7,820 \mathrm{e}$ & 7,768 e & $7,180 \mathrm{~g}$ & $-8,18$ & $82,22 \mathrm{e}$ & $82,66 \mathrm{f}$ & $81,46 \mathrm{f}$ & -0.93 \\
\hline Caipira & $7,806 \mathrm{e}$ & $7,410 \mathrm{e}$ & $7,120 \mathrm{~g}$ & $-9,63$ & $102,55 \mathrm{~b}$ & $102,28 \mathrm{~b}$ & $102,26 \mathrm{~b}$ & -0.28 \\
\hline PV 03-44 & $5,640 \mathrm{f}$ & $5,738 \mathrm{f}$ & $5,558 \mathrm{~h}$ & $-1,47$ & $72,40 \mathrm{f}$ & $72,68 \mathrm{~g}$ & $72,21 \mathrm{~g}$ & -0.26 \\
\hline $\mathrm{CV}$ & $12,64 \%$ & $10,38 \%$ & $10,56 \%$ & & $11,51 \%$ & $10,72 \%$ & $9,66 \%$ & \\
\hline
\end{tabular}

Médias seguidas da mesma letra, na coluna, não diferem entre si pelo teste de SCOTT-KNOTT $(\mathbf{P} \leq 0,05)$ 
TABELA 4 - Valores médios de peso, comprimento e diâmetro de frutos dos três primeiros ciclos de cultivares e híbridos de bananeira. Lavras, MG., 2000.

\begin{tabular}{lccccccccc}
\hline \multirow{2}{*}{$\begin{array}{c}\text { Cultivares/ } \\
\text { Híbridos }\end{array}$} & \multicolumn{3}{c}{ Peso $(\mathbf{g})$} & \multicolumn{4}{c}{ Diâmetro (cm) } & \multicolumn{3}{c}{ Comprimento (cm) } \\
\cline { 2 - 10 } & $\mathbf{1}^{\mathbf{0}}$ Ciclo & $\mathbf{2}^{\mathbf{0}}$ Ciclo & $\mathbf{3}^{\mathbf{0}}$ Ciclo & $\mathbf{1}^{\mathbf{0}}$ Ciclo & $\mathbf{2}^{\mathbf{0}}$ Ciclo & $\mathbf{3}^{\mathbf{0}}$ Ciclo & $\mathbf{1}^{\mathbf{}}$ Ciclo & $\mathbf{2}^{\mathbf{0}}$ Ciclo & $\mathbf{3}^{\mathbf{0}}$ Ciclo \\
\hline SH 36-40 & $157,10 \mathrm{a}$ & $172,02 \mathrm{a}$ & $183,88 \mathrm{a}$ & $4,16 \mathrm{a}$ & $4,22 \mathrm{a}$ & $4,26 \mathrm{a}$ & $16,86 \mathrm{a}$ & $17,84 \mathrm{a}$ & $18,21 \mathrm{a}$ \\
Grande Naine & $131,05 \mathrm{~b}$ & $120,50 \mathrm{c}$ & $119,52 \mathrm{c}$ & $3,88 \mathrm{~b}$ & $3,96 \mathrm{~b}$ & $3,92 \mathrm{c}$ & $15,63 \mathrm{~b}$ & $15,32 \mathrm{c}$ & $15,14 \mathrm{c}$ \\
FHIA 01 & $127,20 \mathrm{c}$ & $134,61 \mathrm{~b}$ & $145,60 \mathrm{~b}$ & $3,88 \mathrm{~b}$ & $3,93 \mathrm{~b}$ & $3,96 \mathrm{~b}$ & $15,57 \mathrm{c}$ & $16,22 \mathrm{~b}$ & $16,60 \mathrm{~b}$ \\
FHIA 18 & $110,35 \mathrm{~d}$ & $113,45 \mathrm{~d}$ & $113,66 \mathrm{~d}$ & $3,87 \mathrm{~b}$ & $3,86 \mathrm{c}$ & $3,87 \mathrm{~d}$ & $14,43 \mathrm{~d}$ & $14,46 \mathrm{~d}$ & $14,45 \mathrm{~d}$ \\
Pioneira & $95,20 \mathrm{e}$ & $95,52 \mathrm{e}$ & $93,52 \mathrm{e}$ & $3,55 \mathrm{~d}$ & $3,55 \mathrm{e}$ & $3,52 \mathrm{f}$ & $13,60 \mathrm{e}$ & $13,62 \mathrm{e}$ & $13,59 \mathrm{e}$ \\
Prata anã & $93,10 \mathrm{e}$ & $95,08 \mathrm{e}$ & $91,02 \mathrm{df}$ & $3,54 \mathrm{~d}$ & $3,58 \mathrm{e}$ & $3,51 \mathrm{f}$ & $13,57 \mathrm{f}$ & $13,61 \mathrm{e}$ & $13,54 \mathrm{fe}$ \\
Nam & $90,71 \mathrm{f}$ & $94,21 \mathrm{e}$ & $95,87 \mathrm{e}$ & $3,61 \mathrm{c}$ & $3,65 \mathrm{~d}$ & $3,67 \mathrm{e}$ & $13,54 \mathrm{~g}$ & $13,56 \mathrm{e}$ & $13,58 \mathrm{fe}$ \\
PV 03-44 & $74,55 \mathrm{~g}$ & $75,40 \mathrm{f}$ & $73,93 \mathrm{~g}$ & $3,32 \mathrm{e}$ & $3,35 \mathrm{f}$ & $3,30 \mathrm{~g}$ & $13,28 \mathrm{~h}$ & $13,26 \mathrm{f}$ & $3,23 \mathrm{def}$ \\
Caipira & $72,26 \mathrm{~g}$ & $72,48 \mathrm{~g}$ & $71,98 \mathrm{~g}$ & $3,25 \mathrm{f}$ & $3,26 \mathrm{~g}$ & $3,22 \mathrm{~h}$ & $12,22 \mathrm{i}$ & $12,21 \mathrm{~g}$ & $12,18 \mathrm{~g}$ \\
\hline CV & $11,88 \%$ & $10,66 \%$ & $11,24 \%$ & $6,84 \%$ & $7,14 \%$ & $7,36 \%$ & $10,42 \%$ & 9,38 & $9,68 \%$ \\
\hline
\end{tabular}

Médias seguidas pela mesma letra, na coluna, não diferem entre si pelo teste de SCOTT-KNOTT $(\mathbf{P} \leq 0,05)$.

De acordo com os dados apresentados na Tabela 3, somente as cultivares Grande Naine e Caipira, nos três ciclos avaliados, produziram cachos com mais de 100 frutos. O Híbrido FHIA 01, somente no terceiro ciclo, ultrapassou 100 frutos por cacho $(103,25)$. Aumento significativo no número de frutos por cacho do primeiro para o terceiro ciclo só foi observado para os híbridos SH 36-40 e FHIA 01 e para a cv. Nam, com incrementos variando entre 5,6 e 7,5\%.

Com relação ao ciclo da cultura (Tabela 5), as cultivares Pioneira e Prata Anã foram as mais precoces, com período total (do plantio a colheita) inferior a 16 meses nos três ciclos ou gerações (plantas - mãe, filha e neta). A cv. Caipira foi a mais tardia, com maiores períodos de pré-floração, (superior a 15 meses), total, acima de 21 meses e de frutificação, juntamente com a cv. Nam, acima de 5,8 meses. O híbrido SH 36-40 apresentou o menor período de frutificação, 4,83 meses no primeiro ciclo e 4,51 no segundo e terceiro ciclos.

Com os períodos de pré-floração e de frutificação mais prolongados, a cv. Caipira apresentou, conseqüentemente, maior período total (do plantio à colheita do cacho) entre 21,5 e 22,7 meses, nos três ciclos. Por outro lado, as cultivares Prata Anã e Pioneira apresentaram os períodos de pré-floração e total inferiores a 12 e 17 meses, respectivamente, no primeiro ciclo, ao passo que no segundo e terceiro ciclos, esses períodos foram inferiores a 11 e 16 meses, em relação aos demais genótipos (Tabela 5). Ganga et al. (2001) também verificaram que essas duas cultivares foram mais precoces no florescimento.

Considerando-se a variação no comprimento de cada período de um ciclo para o outro, verifica-se que esses períodos no primeiro ciclo foram cerca de 1,2 mês maior que no segundo e terceiro ciclos. O período de frutificação, entretanto, apresentou variação bastante irregular de um ciclo para o outro: ora maior do primeiro para o segundo ciclo e menor do segundo para o terceiro, para alguns genótipos, e o contrário para outros. Isso se deve à época de emissão do cacho, que, por sua vez, depende do comprimento do período de pré-floração, que varia de genótipo para genótipo. Desse modo, os cachos emitidos na época fria, com temperaturas inferiores a $15^{\circ} \mathrm{C}$, tiveram o desenvolvimento e grana dos frutos retardados. Essa afirmação fundamenta-se nos relatos de Brunini (1984) e Alves et al. (1999), segundo os quais, temperaturas inferiores a $20^{\circ} \mathrm{C}$ retardam o crescimento das plantas de bananeira e, em Lavras, durante o período de condução do experimento, de maio a agosto, as temperaturas mínimas foram inferiores a $15^{\circ} \mathrm{C}$. 
TABELA 5 - Períodos médios de pré-floração, frutificação e total de cultivares e híbridos de bananeira. Lavras, MG., 2000.

\begin{tabular}{lccccccccc}
\hline & \multicolumn{7}{c}{ Cultivares/ } \\
Híbridos & \multicolumn{3}{c}{ Pré-Floração } & \multicolumn{3}{c}{ Frutificação } & \multicolumn{3}{c}{ Total } \\
\cline { 2 - 10 } & $\mathbf{1}^{\mathbf{0}}$ Ciclo & $\mathbf{2}^{\mathbf{0}}$ Ciclo & $\mathbf{3}^{\mathbf{0}}$ Ciclo & $\mathbf{1}^{\mathbf{0}}$ Ciclo & $\mathbf{2}^{\mathbf{0}}$ Ciclo & $\mathbf{3}^{\mathbf{0}}$ Ciclo & $\mathbf{1}^{\mathbf{0}}$ Ciclo & $\mathbf{2}^{\mathbf{0}}$ Ciclo & $\mathbf{3}^{\mathbf{0}}$ Ciclo \\
\hline Caipira & $16,87 \mathrm{a}$ & $15,85 \mathrm{a}$ & $15,87 \mathrm{a}$ & $5,85 \mathrm{~b}$ & $5,71 \mathrm{~b}$ & $5,78 \mathrm{a}$ & $22,72 \mathrm{a}$ & $21,70 \mathrm{a}$ & $21,56 \mathrm{a}$ \\
Grande Naine & $14,50 \mathrm{~b}$ & $13,52 \mathrm{~b}$ & $13,46 \mathrm{~b}$ & $5,55 \mathrm{c}$ & $5,40 \mathrm{c}$ & $5,18 \mathrm{~b}$ & $20,05 \mathrm{~b}$ & $18,92 \mathrm{~b}$ & $18,74 \mathrm{~b}$ \\
SH 36-40 & $14,05 \mathrm{c}$ & $12,86 \mathrm{c}$ & $12,93 \mathrm{c}$ & $4,83 \mathrm{~g}$ & $4,52 \mathrm{e}$ & $4,50 \mathrm{e}$ & $18,93 \mathrm{~d}$ & $17,53 \mathrm{~d}$ & $17,38 \mathrm{e}$ \\
Nam & $13,52 \mathrm{~d}$ & $12,68 \mathrm{c}$ & $12,66 \mathrm{~d}$ & $6,16 \mathrm{a}$ & $5,86 \mathrm{a}$ & $5,85 \mathrm{a}$ & $19,68 \mathrm{c}$ & $18,54 \mathrm{c}$ & $18,51 \mathrm{c}$ \\
PV 03-44 & $13,48 \mathrm{~d}$ & $12,64 \mathrm{c}$ & $12,42 \mathrm{e}$ & $5,50 \mathrm{~d}$ & $5,31 \mathrm{c}$ & $5,25 \mathrm{~b}$ & $18,92 \mathrm{~d}$ & $17,75 \mathrm{~d}$ & $17,67 \mathrm{~d}$ \\
FHIA 18 & $13,40 \mathrm{~d}$ & $12,44 \mathrm{~d}$ & $12,40 \mathrm{e}$ & $5,53 \mathrm{~d}$ & $5,34 \mathrm{c}$ & $5,21 \mathrm{~b}$ & $18,93 \mathrm{~d}$ & $17,72 \mathrm{~d}$ & $17,61 \mathrm{~d}$ \\
FHIA 01 & $13,36 \mathrm{~d}$ & $12,38 \mathrm{~d}$ & $12,28 \mathrm{f}$ & $5,51 \mathrm{~d}$ & $5,32 \mathrm{c}$ & $5,30 \mathrm{~b}$ & $18,87 \mathrm{~d}$ & $17,63 \mathrm{~d}$ & $17,60 \mathrm{~d}$ \\
Prata anã & $11,82 \mathrm{e}$ & $10,77 \mathrm{e}$ & $10,75 \mathrm{~g}$ & $5,17 \mathrm{e}$ & $5,04 \mathrm{~d}$ & $4,76 \mathrm{~d}$ & $16,99 \mathrm{e}$ & $15,90 \mathrm{e}$ & $15,50 \mathrm{f}$ \\
Pioneira & $11,06 \mathrm{f}$ & $10,08 \mathrm{f}$ & $10,02 \mathrm{~h}$ & $5,31 \mathrm{e}$ & $5,30 \mathrm{~d}$ & $5,06 \mathrm{c}$ & $16,35 \mathrm{f}$ & $15,38 \mathrm{f}$ & $15,08 \mathrm{e}$ \\
\hline CV & $10,44 \%$ & $11,26 \%$ & $10,74 \%$ & $8,56 \%$ & $7,28 \%$ & $7,78 \%$ & $9,86 \%$ & $9,32 \%$ & $8,90 \%$ \\
\hline
\end{tabular}

Médias seguidas pela mesma letra, na coluna, não diferem entre si pelo teste de SCOOTT-KNOTT $(\mathbf{P} \leq 0,05)$.

As diferenças no índice de perfilhamento (número de filhos e netos) observadas entre os híbridos e cultivares avaliados neste trabalho são confirmados pelos relatos de Souza et al.(1996); Lameira et al. (1988) e Dantas et al. (1986). Segundo esses autores, diferenças na capacidade de proliferação de cada genótipo são evidentes, afetando, assim, a produção de rebentos. Silva \& Alves (1999) também encontraram bom índice de perfilhamento nas cultivares Caipira, Prata Anã e Pioneira e nos híbridos FHIA 01 e FHIA 18. Entretanto, neste trabalho, esses dois híbridos e a cv. Grande Naine apresentaram menor índice de perfilhamento que aquelas cultivares.

O melhor comportamento produtivo dos híbridos SH 36-40 e FHIA 01 e da cv. Grande Naine verificado neste trabalho, em Lavras, MG, vem confirmar a melhor performance produtiva desses genótipos em outros locais, como no perímetro irrigado da região norte de Minas Gerais e em Cruz das Almas, BA (Gonçalves et al., 2001; Rodrigues et al., 2001; Souto et al., 2001; Silva \& Alves, 1999).

A maior produção do híbrido $\mathrm{SH} 36-40 \mathrm{em}$ condições de sequeiro nos três ciclos avaliados, com cachos cerca de duas vezes mais pesados (Tabela 3) que as cultivares Prata Anã, Pioneira, Caipira e Nam e que o híbrido PV 03-44, vem confirmar os resultados obtidos por Silva \& Alves (1999) em condições de sequeiro na
Bahia e por Gonçalves et al. (2001), Souto et al.(2001) e Rodrigues et al. (2001) em condições irrigadas na região norte de Minas Gerais.

Em Cruz das Almas, BA, o híbrido FHIA 01 e a cv. Grande Naine produziram, também, cachos cerca de duas vezes mais pesados que as cultivares Prata Anã, Pioneira, Nam e o híbrido PV 03-44 (Silva \& Alves, 1999). Em Lavras, somente no primeiro ciclo, a cv. Grande Naine produziu cachos com peso semelhante ao do híbrido SH 36-40, e maior que o do híbrido FHIA 01. No segundo e, principalmente, no terceiro ciclo, entretanto, esses dois híbridos produziram cachos e frutos significativamente maiores que a cv. Grande Naine, resultados esses semelhantes aos de Alvarez (1997), em Cuba, e de Navarro \& Caicedo (1998) na Colombia, onde a produção do híbrido FHIA 01 foi cerca de duas vezes maior que a da cv. Grande Naine.

Com relação ao tamanho de fruto, os resultados desse trabalho são semelhantes àqueles de Cerqueira et al. (2001), os quais relataram que o híbrido SH 36-40 produziu frutos maiores que as cultivares Prata Anã, Prata, Pacovan, Nam e que o híbrido PV 03-44.

O maior incremento na produção (peso e tamanho) do cacho e dos frutos dos híbridos SH 36-40 e FHIA 01, do primeiro para o segundo e deste para o terceiro ciclo, pode ser devido, provavelmente, às caracte- 
rísticas genéticas desses genótipos, a maior tolerância ao déficit hídrico, à Sigatoka amarela e ao frio.

As condições climáticas e principalmente as características genéticas de cada genótipo têm influência significativa no ciclo da cultura (Silva et al., 1999). Isso foi constatado com as cultivares Pioneira e Prata Anã, que apresentaram maior precocidade que as cultivares Caipira e Nam e os híbridos FHIA 01, FHIA18, SH 3640 e PV 03-44 neste trabalho em Lavras-MG e também em Jaíba-MG (Gonçalves et al., 2001), Cruz das AlmasBA (Silva \& Alves, 1999), Guanambi e Cruz das Almas-BA, Viçosa, Lavras e Mocambinho. Por outro lado, Silva et al. (2001) observaram que os híbridos FHIA 18 e PA03-22 foram mais precoces em Cruz das Almas que as cultivares Pioneira, Nam, Grande Naine, Caipira, Prata e Prata Anã.

Ficou evidenciada a influência da irrigação no ciclo da cultura, uma vez que as cultivares Prata Anã, Pioneira, Caipira, FHIA 01, FHIA 18 e SH 36-40, sob condições irrigadas em Jaíba (MG), foram mais precoces que sob condições de sequeiro, cerca de 6,6 meses em relação a Lavras (MG) e 1,2 mês em relação a Cruz das Almas (BA).

As diferenças na precocidade dessas cultivares nos três locais, independentemente da irrigação, e o maior ciclo da cultura em Lavras, são atribuídos ao clima mais frio de Lavras, com temperatura mínima em torno de $15,3^{\circ} \mathrm{C}$, bastante inferior à de Cruz das Almas e Jaíba. Esse argumento fundamenta-se nas afirmações de Brunini (1984) e Alves et al. (1999), segundo os quais temperaturas inferiores a $20^{\circ} \mathrm{C}$ retardam o desenvolvimento da bananeira.

\section{CONCLUSÕES}

O híbrido SH 36-40 foi superior aos demais genótipos, considerando-se o peso médio dos cachos e dos frutos, diâmetro e comprimento dos frutos, ciclo e porte médios e perfilhamento mais tardio e menos intenso.

O híbrido PV 03-44 e a cv. Caipira foram os menos produtivos, além de apresentarem porte alto.

Os maiores incrementos nos pesos dos cachos e dos frutos e no número de frutos por cacho, de um ciclo para o outro, foram observados para os híbridos SH 3640 e FHIA 01.

\section{REFERÊNCIAS BIBLIOGRÁFICAS}

ALVAREZ, Z. M. Introdución, evaluación, multiplicación y disseminación de híbridos FHIA en Cuba. Infomusa, Montpellier, n. 2, p. 10-14, 1997.
ALVES, E. J.; OLIVEIRA, M. de A.; DANTAS, J. L. L.; OLIVEIRA, S. L. de. Exigências climáticas. In: ALVES, E. J. A cultura da bananeira: aspectos técnicos, sócioeconômicos e agroindustriais. 2. ed. Brasília: EMBRAPA/SPI, 1999. Cap. 5, p. 85-105.

BRUNINI, O. Exigências climáticas e aptidão agroclimática da bananicultura. In: SIMPÓSIO BRASILEIRO SOBRE BANANICULTURA, 1., 1984.; Fortaleza. Anais... Jaboticabal: FCAVJ, 1984. p. 99-117.

CERQUEIRA, R. C.; SILVA, S. de O.; MEDINA, V. M. Características da maturação pós-colheita e resistência ao despencamento de genótipos de bananeira (Musa spp.). In: CONGRESSO BRASILEIRO DE FRUTICULTURA, 16., 2001, Fortaleza. Resumos: Fortaleza: SBF/EMBRAPA, 2001. p. 119.

COMISSÃO DE FERTILIDADE DO SOLO DO ESTADO DE MINAS GERAIS. Recomendações para o uso de corretivos e fertilizantes em Minas Gerais: $4^{\mathrm{a}}$ aproximação. Lavras, 1989. 176 p.

DANTAS, J. L. L.; SHEPHERD, K.; ALVES, E. J. Propagação rápida da bananeira. Informe Agropecuário, Belo Horizonte, v. 12, n. 137, v. 33-38, 1986.

FERREIRA, D. F. Análises estatísticas por meio do SISVAR para Windows versão 3.0. In: REUNIÃO ANUAL DA REGIÃO BRASILEIRA DA SOCIEDADE INTERNACIONAL DE BIOMETRIA, 45., 2000, São Carlos. Programas e Resumos... São Carlos: UFSCar, 2000. p. 233.

FRUTISÉRIES. Banana. Brasília: Ministério da Integração Nacional, 2000. _ 8 p. Disponível em: 'Lttp://<www.integração.gov.bry. Acesso em: out. 2000.

GANGA, R. M. D.; RUGGIERO, C.; MARTINS, A. B. G. Comportamento dos cultivares de bananeiras Prata Anã, Pioneira, Maça, Nanicão, FHIA 01 e FHIA 18 nas condições de Jaboticabal/SP. In: CONGRESSO BRASILEIRO DE FRUTICULTURA, 16., 2000, Fortaleza. Resumos... Fortaleza: SBF/EMBRAPA, 2001. p. 109.

GOMEZ, J. A.; NOBREGA, A. C. Comportamento de cultivares e híbridos de bananeira na região produtora do Estado do Espírito Santo. Revista Brasileira de Fruticultura, Jaboticabal, v. 22, n. 1, p. 11-13, abr. 2000. 
GONÇALVES, M. I. F.; RODRIGUES, M. G. V.; SOUTO, R. F.; SILVA, S. de O. e; MENEGUCCI, J. L. P. Avaliação de genótipos de bananeira cultivadas no Projeto Jaíba, Região Norte de Minas Gerais. In. CONGRESSO BRASILEIRO DE FRUTICULTURA, 16., 2001, Fortaleza. Resumos... Fortaleza: SBF/EMBRAPA, 2001. p. 118.

LAMEIRA, O. A.; PINTO, J. E. B. P.; PASQUAL, M. Efeito do tamanho do explante no desenvolvimento "in vitro" da bananeira (musa acuminata Colla). Cultivares Prata e Nanicão. Ciência e Prática, Lavras, v. 12, n. 2, p. 207-211, 1988.

NAVARRO, E. E.; CAICEDO, L. E. G. Evaluación de híbridos y clones de plátano y banana tolerantes a la Sigatoka negra en el Centro-Sur del Departamento del Tolima, Colômbia. Infomusa, Montpellier, v. 7, n. 2, p. 14-16, 1998.

ORJEDA, G.; ESCALANT, J. V.; MOORE, N. Programa internacional de evaluación de Musa (IMTP) fase II. Sinopsis del informe final y resumen de los resultados. Infomusa, Montpellier, v. 8, n. 1, p. 3-10, 1999.

PEREIRA, L. V.; REZENDE E SILVA, C. R. de; ALVARENGA, A. A. Comportamento de cultivares de bananeira oriundas de mudas de cultura de tecido e convencionais. In: CONGRESSO BRASILEIRO DE FRUTICUlTURA, 15., 1998, Poços de Caldas. Resumos... Lavras: UFLA, 1998. p. 125.

RODRIGUES, M. G., SOUTO, F. S., SILVA, S. de O. e; MENEGUCCI, J. L. P. Avaliação de genótipos de bananeira cultivados no Projeto Jaíba, Região Norte de Minas Gerais. In: SIMPÓSIO BRASILEIRO SOBRE BANANICULTURA, 4, 1998, Jaboticabal. Anais... Jaboticabal: FUNEP, 2001. p. 523.

SILVA, S. de O. e ALVES, E. J. Melhoramento genético e novas cultivares de bananeira. Informe Agropecuário, Belo Horizonte, v. 20, n. 196, p. 91-96, jan./fev. 1999

SILVA, S. de O.; ALVES, E. J.; SHEPHERD, K.; DANTAS, Z. L. L. Cultivares. In: ALVES, E. J. A cultura da banana: aspectos técnicos, sócioeconômi- cos e agroindustriais. 2. ed. Brasília: EMBRAPA/SPI/, 1999. Cap. 5, p. 85-105.

SILVA, S. de O.; FLORES, C. de O.; LIMA NETO, F. P. Avaliação de quatro ciclos de cultivares e híbridos de bananeira em Cruz das Almas - BA. In: CONGRESSO BRASILEIRO DE FRUTICULTURA, 16., 2000, Fortaleza. Resumos... Fortaleza: SBF/EMBRAPA, 2000. p. 113.

SILVEIRA, J. R. S.; SOUTO, R. F.; ALVES, E. J.; OLIVEIRA, M. de A.; OLIVEIRA, E.; SILVA, S. de. Avaliação agronômica de cultivares e híbridos de banana de porte baixo na Região Norte de Minas Gerais. In: CONGRESSO BRASILEIRO DE FRUTICULTURA, 14., 1996, Curitiba. Resumos... Londrina: IAPAR, 1996. p. 82.

SOUTO, R. F.; ALVES, E. J.; SILVA, S. de O.; SILVERIA, J. R. S.; OLIVEIRA, M. de A. Avaliação agronômica de cultivares e híbridos de banana de porte alto na Região Norte de Minas Gerais. In: CONGRESSO BRASILEIRO DE FRUTICULTURA, 14., 1996, Curitiba. Resumos... Londrina: IAPAR, 1996. p. 81.

SOUTO, F. R.; RODRIGUES, M. G. V.; SILVA, S. de O.; MENEGUCCI, J. L. P. Avaliação de genótipos de bananeira cultivadas no Projeto Gorutuba, Região Norte de MINAS GERAIS. In: SIMPÓSIO BRASILEIRO SOBRE BANANICULTURA, 4., 1998, Jaboticabal. Anais... Jaboticabal: FUNEP, 2001. p. 519.

SOUZA, J. L. L.; SHEPHERD, K.; ALVES, E. J. Propagação rápida da bananeira. Informe Agropecuário, Belo Horizonte, v. 12, n. 133, p. 33-38, jan. 1996. 